\title{
COMBINED PROTON MAGNETIC RESONANCE SPECTROSCOPY AND NEAR-INFRARED SPECTROSCOPY MEASUREMENTS OF CEREBRAL BLOOD VOLUME, OXYGENATION, CYTOCHROME OXIDASE, AND INTRACELLULAR METABOLITES DURING PERINATAL HYPOXIA-ISCHAEMIA
}

\author{
E. Powell ${ }^{1}$, S. Faulkner ${ }^{1}$, I. Tachtsidis ${ }^{2}$, A. Bainbridge ${ }^{3}$, D. Price ${ }^{3}$, C. Andorka ${ }^{1}$, S. Mahony ${ }^{2}$, L. Lecky- \\ Thompson ${ }^{1}$, E. Cady $^{3}$, X. Golay ${ }^{4}$, N.J. Robertson ${ }^{1}$ \\ ${ }^{1}$ Institute for Women's Health, ${ }^{2}$ Department of Medical Physics and Bioengineering, University College \\ London, ${ }^{3}$ Medical Physics and Bioengineering, University College London Hospital NHS Foundation Trust, \\ ${ }^{4}$ Institute of Neurology, University College London, London, UK
}

Background: Hypoxic-ischaemic (HI) neonatal encephalopathy (NE) is associated with high mortality and morbidity rates worldwide. The magnetic resonance spectroscopy (MRS) Lactate/ $\mathrm{N}$-acetylaspartate (Lac/NAA) peak-area ratio predicts long term neurodevelopmental outcome.

Aims: To investigate brain haemodynamic and metabolic changes during transient HI using simultaneous proton (1H) MRS and broadband near-infra-red spectroscopy (NIRS) in a newborn piglet pre-clinical model.

Methods: MRS and NIRS data were acquired simultaneously in 6 healthy newborn anaesthetised male piglets (aged $<24 \mathrm{hr}$ ) during, and up to $90 \mathrm{~min}$ after transient HI.

Results: A swift decline in oxygenation (Hbdiff) upon induction of $\mathrm{HI}$, and associated reduction of cytochrome-c-oxidase (ox-red CCO), was accompanied by increased Lac/Naa (Fig 1A-D). Upon resuscitation there was a hyperaemic phase in 4 out of 6 animals with ox-red $\mathrm{CCO}$ and Lac/Naa recovering to baselines. During recovery ox-red $\mathrm{CCO}$ and Lac/Naa correlated linearly ( $\mathrm{p}=<0.0001$; Pearson correlation) (Fig. 1D). Two piglets showed no post $\mathrm{HI}$ hyperaemic response and incomplete ${ }_{\text {ox-red }} \mathrm{CCO}$ recovery.

Conclusions: The linear relationship between declining Lac/Naa and increasing ox-red $\mathrm{CCO}$ after $\mathrm{HI}$ suggests mitochondrial utilisation of Lac, instead of pyruvate, as a substrate at least during oxidative energy metabolism recovery. Complementary 1H MRS and NIRS may improve our understanding of cerebral energy metabolism pathways and the response of the newborn brain to HI and govern early interventional therapies.

A
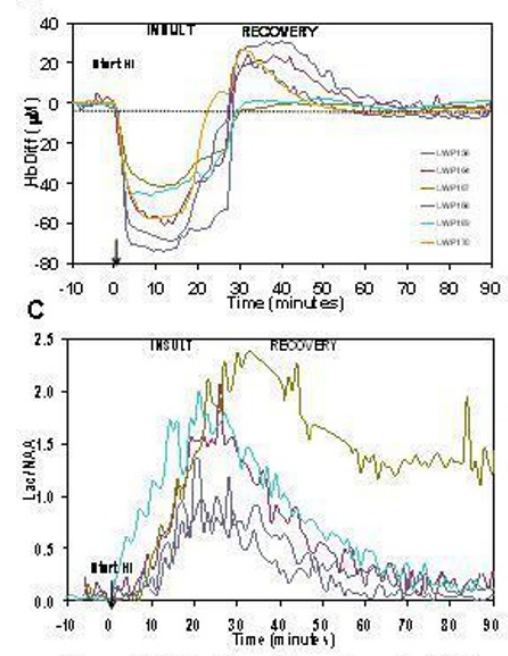

B
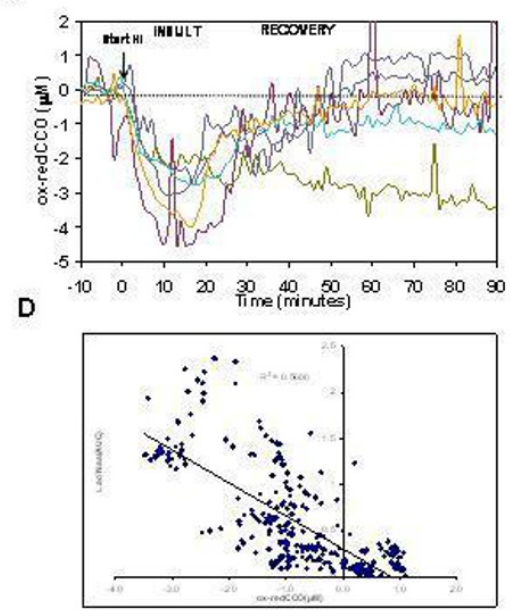

Figure 1 Relationship of haemodynamic (HIRS) and MRS $1 \mathrm{H}$ Lac/Naa changes during an hypoxic

ischeremic insult and recovery. A-C; changes in oxygenation (HbDiff), ox-red $\mathrm{CCO}$ and Lac/Naa during insulk and recovery. D; Comelation of ox-red $\mathrm{CCO}$ us LacNaa during recovery phase

[Figure 1 Relationship of haemodynamic (NIRS) and] 